\title{
GLOBAL WELL-POSEDNESS OF THE MHD EQUATIONS VIA THE COMPARISON PRINCIPLE
}

\author{
DONGYI WEI AND ZHIFEI ZHANG
}

\begin{abstract}
In this paper, we prove the global well-posedness of the incompressible MHD equations near a homogeneous equilibrium in the domain $\mathbb{R}^{k} \times \mathbb{T}^{d-k}, d \geq 2, k \geq 1$ by using the comparison principle and constructing the comparison function.
\end{abstract}

\section{INTRODUCTION}

In this paper, we consider the incompressible magneto-hydrodynamics (MHD) equations in $[0, T) \times \Omega, \Omega \subseteq \mathbb{R}^{d}, d \geq 2$ :

$$
\left\{\begin{array}{l}
\partial_{t} v-\nu \Delta v+v \cdot \nabla v+\nabla p=b \cdot \nabla b \\
\partial_{t} b-\mu \Delta b+v \cdot \nabla b=b \cdot \nabla v \\
\operatorname{div} v=\operatorname{div} b=0
\end{array}\right.
$$

where $v$ denotes the velocity field and $b$ denotes the magnetic field, and $\nu \geq 0$ is the viscosity coefficient, $\mu \geq 0$ is the resistivity coefficient. If $\nu=\mu=0$, (1.1) is the so called ideal MHD equations; If $\nu>0$ and $b=0$, (1.1) is reduced to the Navier-Stokes equations.

It is well-known that the 2-D MHD equations with full viscosities(i.e., $\nu>0$ and $\mu>0$ ) have global smooth solution. In the absence of resistivity(i.e., $\mu=0$ ), the global existence of weak solution and strong solution of the MHD equations is still an open question. Recently, Cao and $\mathrm{Wu}[5$ ] proved the global regularity of the 2-D MHD equations with partial dissipation and magnetic diffusion. Motivated by numerical observation [3]: the energy of the MHD equations is dissipated at a rate independent of the ohmic resistivity, there are a lot of works [1, 17, 8, 9, 11] devoted to the global well-posedness of the MHD equations without resistivity in a homogeneous magnetic field $B_{0}$.

In high temperature plasmas, both $\nu$ and $\mu$ are usually very small. Thus, it is very interesting to investigate the long-time dynamics of the MHD equations in such case. In this paper, we consider the case of $\mu=\nu$. In terms of the Elsässer variables

$$
Z_{+}=v+b, \quad Z_{-}=v-b,
$$

the MHD equations (1.1) can be written as

$$
\left\{\begin{array}{l}
\partial_{t} Z_{+}+Z_{-} \cdot \nabla Z_{+}=\mu \Delta Z_{+}-\nabla p \\
\partial_{t} Z_{-}+Z_{+} \cdot \nabla Z_{-}=\mu \Delta Z_{-}-\nabla p \\
\operatorname{div} Z_{+}=\operatorname{div} Z_{-}=0
\end{array}\right.
$$

We introduce the fluctuations

$$
z_{+}=Z_{+}-B_{0}, \quad z_{-}=Z_{-}+B_{0} .
$$

Date: October 8, 2018. 
Then the system (1.2) can be reformulated as

$$
\left\{\begin{array}{l}
\partial_{t} z_{+}+Z_{-} \cdot \nabla z_{+}=\mu \Delta z_{+}-\nabla p \\
\partial_{t} z_{-}+Z_{+} \cdot \nabla z_{-}=\mu \Delta z_{-}-\nabla p \\
\operatorname{div} z_{+}=\operatorname{div} z_{-}=0 .
\end{array}\right.
$$

Bardos-Sulem-Sulem [2] proved the global well-posedness of (1.3) for $\Omega=\mathbb{R}^{d}$ and $\mu=0$ when the initial data is small in a weighed Hölder space. Recently, He-Xu-Yu [6] proved the global well-posedness of (1.3) for any $\mu \geq 0$ and $\Omega=\mathbb{R}^{3}$ by using some ideas from nonlinear stability of Minkowski space-time in general relativity. Cai and Lei [4] proved simliar result for $\Omega=\mathbb{R}^{d}, d=2,3$ by using Alinhac's ghost weight method. Wei and Zhang [10] dealt with more physical case, which allows $\nu \neq \mu$ and $\Omega$ to be a strip. For all these results, the key mechanism leading to the global well-posedness is that the nonlinear terms $z_{-} \cdot \nabla z_{+}$and $z_{+} \cdot \nabla z_{-}$are essentially neglected after a long time, because $z_{ \pm}$are transported along the opposite direction.

The goal of this paper is twofold: (1) include the domain $\mathbb{R}^{k} \times \mathbb{T}^{d-k}, k \geq 1$. Previous results required $k \geq 2$ at least; (2) develop an elementary and much simpler method via the comparison principle.

Without loss of generality, we take the background magnetic field $B_{0}=(1,0, \cdots, 0)$. We will identify a function in $\mathbb{R}^{k} \times \mathbb{T}^{d-k}$ with a function in $\mathbb{R}^{d}$ periodic in $d-k$ directions $e_{1}, \cdots, e_{d-k}$, where $e_{1}, \cdots, e_{d-k}, B_{0}$ are orthogonal.

We introduce

$$
\rho_{ \pm}(t, X)^{2}=\sum_{k=0}^{N} \int_{\mathbb{R}^{d}}\left|\nabla^{k} z_{ \pm}\right|^{2}(t, Y) \theta(|X-Y|) d Y,
$$

where $\theta(r)$ is a smooth cut-off function so that

Let

$$
\theta(r)=\left\{\begin{array}{ll}
1 & \text { for }|r| \leq 1, \\
0 & \text { for }|r| \geq 2,
\end{array} \quad\left|\theta^{\prime}(r)\right|^{2} \leq C \theta(r) .\right.
$$

Our result is stated as follows.

Theorem 1.1. Let $d \geq 2,1 \leq k \leq d$ and $z_{ \pm}(0) \in H^{N}\left(\mathbb{R}^{k} \times \mathbb{T}^{d-k}\right)$ for some integer $N>\frac{d}{2}+1$. There exists $\epsilon_{1}>0$ so that if $J_{ \pm}(0) \leq \epsilon_{1}$, then there exists a global unique solution $\left(z_{+}, z_{-}\right) \in$ $C\left([0,+\infty), H^{N}\left(\mathbb{R}^{k} \times \mathbb{T}^{d-k}\right)\right)$ to the MHD equations (1.3) satisfying

$$
\left\|z_{ \pm}(t)\right\|_{H^{N}\left(\mathbb{R}^{k} \times \mathbb{T}^{d-k}\right)} \leq C\left\|z_{ \pm}(0)\right\|_{H^{N}\left(\mathbb{R}^{k} \times \mathbb{T}^{d-k}\right)} \quad \text { for any } t \in[0,+\infty) .
$$

Let us give some remarks on our result.

1. We only require the initial data to decay at infinity in $B_{0}$ direction, which is the key for the global well-posedness in $\mathbb{R} \times \mathbb{T}^{d-1}$.

2. It is easy to check that $J_{ \pm}(0) \leq C \epsilon$ for the initial data considered in [4] and [6]. Indeed, for the data in [4], we have $\rho_{ \pm}(0, X) \leq C \epsilon(1+|X|)^{-\delta}$. For the data in [6], we have $\rho_{ \pm}(0, X) \leq C \epsilon\left(R^{2}+|X|^{2}\right)^{-\frac{1}{2}}\left(\ln \left(R^{2}+|X|^{2}\right)\right)^{-2}$. Here $\delta>1, R \geq 100$ and $\epsilon>0$ is small.

3. At a first glance, there seems no difference between $\mathbb{R}^{d}$ and $\mathbb{R}^{k} \times \mathbb{T}^{d-k}$. From the proofs in [4] and 6], it seems that the case of $d=3$ is easier than the case of $d=2$. However, for a solution $\left(z_{+}, z_{-}\right) \in C\left([0,+\infty), H^{N}\left(\mathbb{R}^{2}\right)\right),\left(z_{+}(t, x), 0, z_{-}(t, x), 0\right)$ is also a solution in $C\left([0,+\infty), H^{N}\left(\mathbb{R}^{2} \times \mathbb{T}\right)\right)$. Thus, the case of $\mathbb{R}^{2} \times \mathbb{T}$ is not easier than the case of $\mathbb{R}^{2}$. In this sense, the case of $\mathbb{R} \times \mathbb{T}^{d-1}$ may be harder. 
Throughout this paper, we denote by $C$ a constant independent of $t, \mu$, which may be different from line to line.

\section{LOCAL ENERGY INEQUALITY}

In this section, we derive the following local energy inequalities

$$
\partial_{t} \rho_{ \pm} \mp B_{0} \cdot \nabla \rho_{ \pm}-\mu \triangle \rho_{ \pm} \leq C F,
$$

where $F(t, X)$ is given by

$$
(2.2) F(t, X)=\sum_{k+j \leq N+1,0 \leq k, j \leq N}\left\|\left|\nabla^{k} z_{+}\left\|\nabla^{j} z_{-} \mid(t)\right\|_{L^{2}(B(X, 2))}+\sum_{k=0}^{N}\left\|\nabla^{k+1} p(t)\right\|_{L^{2}(B(X, 2))} .\right.\right.
$$

Here $B(X, r)$ denotes a ball in $\mathbb{R}^{d}$ with the center at $X$ and radius $r$.

We only prove (2.1) for $\rho_{+}$, the case of $\rho_{-}$is similar. For any multi-index $a \in \mathbb{N}^{d}$, using the same notations as in [4, one can easily deduce from (1.3) that

$$
\left\{\begin{array}{l}
\partial_{t} \nabla^{a} z_{+}-\mu \Delta \nabla^{a} z_{+}-B_{0} \cdot \nabla \nabla^{a} z_{+}+\sum_{b+c=a} C_{a}^{b}\left(\nabla^{b} z_{-} \cdot \nabla \nabla^{c} z_{+}\right)+\nabla \nabla^{a} p=0 \\
\partial_{t} \nabla^{a} z_{-}-\mu \Delta \nabla^{a} z_{-}+B_{0} \cdot \nabla \nabla^{a} z_{-}+\sum_{b+c=a} C_{a}^{b}\left(\nabla^{b} z_{+} \cdot \nabla \nabla^{c} z_{-}\right)+\nabla \nabla^{a} p=0 \\
\operatorname{div} \nabla^{a} z_{+}=\operatorname{div} \nabla^{a} z_{-}=0 .
\end{array}\right.
$$

Taking inner product of the first equation with $\nabla^{a} z_{+}$, we obtain

$$
\begin{aligned}
\partial_{t}\left|\nabla^{a} z_{+}\right|^{2} & -\mu \Delta\left|\nabla^{a} z_{+}\right|^{2}+2 \mu\left|\nabla \nabla^{a} z_{+}\right|^{2}-B_{0} \cdot \nabla\left|\nabla^{a} z_{+}\right|^{2} \\
& +2 \sum_{b+c=a} C_{a}^{b}\left(\nabla^{b} z_{-} \cdot \nabla \nabla^{c} z_{+} \nabla^{a} z_{+}\right)+2 \nabla^{a} z_{+} \cdot \nabla \nabla^{a} p=0,
\end{aligned}
$$

from which, we deduce that

$$
\begin{array}{r}
\left(\partial_{t} \rho_{+}^{2}-\mu \triangle \rho_{+}^{2}-B_{0} \cdot \nabla \rho_{+}^{2}\right)(t, X)+2 \mu \int_{\mathbb{R}^{d}} \sum_{|a| \leq N}\left|\nabla \nabla^{a} z_{+}\right|^{2}(t, Y) \theta(|X-Y|) d Y \\
+2 \int_{\mathbb{R}^{d}} \sum_{|a| \leq N} \sum_{b+c=a} C_{a}^{b}\left(\nabla^{b} z_{-} \cdot \nabla \nabla^{c} z_{+} \nabla^{a} z_{+}\right)(t, Y) \theta(|X-Y|) d Y \\
+2 \int_{\mathbb{R}^{d}} \sum_{|a| \leq N} \nabla^{a} z_{+} \cdot \nabla \nabla^{a} p(t, Y) \theta(|X-Y|) d Y=0 .
\end{array}
$$

Let $\rho_{+}^{(\varepsilon)}=\left(\rho_{+}^{2}+\varepsilon\right)^{\frac{1}{2}}$ for $\varepsilon>0$. We have

$$
\begin{aligned}
& 2\left(\rho_{+}^{(\varepsilon)} \nabla \rho_{+}^{(\varepsilon)}\right)(t, X)=\left(\nabla \rho_{+}^{2}\right)(t, X)=2 \int_{\mathbb{R}^{d}} \sum_{|a| \leq N}\left(\nabla \nabla^{a} z_{+} \cdot \nabla^{a} z_{+}\right)^{2}(t, Y) \theta(|X-Y|) d Y \\
& \leq 2\left(\int_{\mathbb{R}^{d}} \sum_{|a| \leq N}\left|\nabla \nabla^{a} z_{+}\right|^{2}(t, Y) \theta(|X-Y|) d Y \int_{\mathbb{R}^{d}} \sum_{|a| \leq N}\left|\nabla^{a} z_{+}\right|^{2}(t, Y) \theta(|X-Y|) d Y\right)^{\frac{1}{2}} \\
& =2\left(\int_{\mathbb{R}^{d}} \sum_{|a| \leq N}\left|\nabla \nabla^{a} z_{+}\right|^{2}(t, Y) \theta(|X-Y|) d Y\right)^{\frac{1}{2}} \rho_{+}(t, X),
\end{aligned}
$$


which implies that

$$
\int_{\mathbb{R}^{d}} \sum_{|a| \leq N}\left|\nabla \nabla^{a} z_{+}\right|^{2}(t, Y) \theta(|X-Y|) d Y \geq\left|\nabla \rho_{+}^{(\varepsilon)}(t, X)\right|^{2} .
$$

If $|a| \leq N, b+c=a, c<a$, then $|b|,|c|+1 \leq N$ and

$$
\begin{aligned}
& -\int_{\mathbb{R}^{d}}\left(\nabla^{b} z_{-} \cdot \nabla \nabla^{c} z_{+} \nabla^{a} z_{+}\right)(t, Y) \theta(|X-Y|) d Y \\
& \leq\left(\int_{B(X, 2)}\left|\nabla^{|b|} z_{-}\right|^{2}\left|\nabla^{|c|+1} z_{+}\right|^{2}(t, Y) d Y \int_{\mathbb{R}^{d}}\left|\nabla^{a} z_{+}\right|^{2}(t, Y) \theta(|X-Y|) d Y\right)^{\frac{1}{2}} \\
& \leq F(t, X) \rho_{+}(t, X) .
\end{aligned}
$$

If $|a| \leq N, b+c=a, c=a$, then $b=(0, \cdots, 0)$ and

$$
\begin{aligned}
& -2 \int_{\mathbb{R}^{d}}\left(\nabla^{b} z_{-} \cdot \nabla \nabla^{c} z_{+} \nabla^{a} z_{+}\right)(t, Y) \theta(|X-Y|) d Y \\
& =-\int_{\mathbb{R}^{d}}\left(z_{-} \cdot \nabla\left|\nabla^{a} z_{+}\right|^{2}\right)(t, Y) \theta(|X-Y|) d Y \\
& =-\int_{\mathbb{R}^{d}} z_{-} \cdot \nabla \theta(|X-Y|)\left|\nabla^{a} z_{+}\right|^{2}(t, Y) d Y \\
& \leq C\left(\int_{B(X, 2)}\left|z_{-}\right|^{2}\left|\nabla^{|a|} z_{+}\right|^{2}(t, Y) d Y \int_{\mathbb{R}^{d}}\left|\nabla^{a} z_{+}\right|^{2}(t, Y) \theta(|X-Y|) d Y\right)^{\frac{1}{2}} \\
& \leq C F(t, X) \rho_{+}(t, X) .
\end{aligned}
$$

If $|a| \leq N$, then

$$
\begin{aligned}
& -\int_{\mathbb{R}^{d}} \nabla^{a} z_{+} \cdot \nabla \nabla^{a} p(t, Y) \theta(|X-Y|) d Y \\
& \leq\left(\int_{B(X, 2)}\left|\nabla^{|a|+1} p\right|^{2}(t, Y) d Y \int_{\mathbb{R}^{d}}\left|\nabla^{a} z_{+}\right|^{2}(t, Y) \theta(|X-Y|) d Y\right)^{\frac{1}{2}} \\
& \leq F(t, X) \rho_{+}(t, X) .
\end{aligned}
$$

Summing up, we obtain

$$
\left(\partial_{t} \rho_{+}^{2}-\mu \triangle \rho_{+}^{2}-B_{0} \cdot \nabla \rho_{+}^{2}\right)(t, X)+2 \mu\left|\nabla \rho_{+}^{(\varepsilon)}(t, X)\right|^{2} \leq C F(t, X) \rho_{+}(t, X),
$$

which gives

$$
\left(\partial_{t} \rho_{+}^{(\varepsilon)}-\mu \triangle \rho_{+}^{(\varepsilon)}-B_{0} \cdot \nabla \rho_{+}^{(\varepsilon)}\right)(t, X) \leq C F(t, X) .
$$

Now, the local energy inequality (2.1) follows by letting $\varepsilon \rightarrow 0$.

Let us conclude this section by the following estimate for $F(t, X)$ :

$$
F(t, X) \leq C \int_{\mathbb{R}^{d}} \frac{\rho_{+}(t, Y) \rho_{-}(t, Y)}{1+|X-Y|^{d+1}} d Y .
$$

We need the following fact.

Lemma 2.1. It holds that

$$
\|f\|_{L^{2}(B(X, 2))} \leq C \int_{B(X, 3)}\|f\|_{L^{2}\left(B\left(Y, \frac{1}{2}\right)\right)} d Y .
$$


Proof. By Fubini theorem, we have

$$
\begin{aligned}
\int_{B\left(X, \frac{1}{2}\right)}\|f\|_{L^{2}(B(X, 2) \cap B(Y, 3))}^{2} d Y & =\int_{B(X, 3)}\|f\|_{L^{2}\left(B(X, 2) \cap B\left(Y, \frac{1}{2}\right)\right)}^{2} d Y \\
& \leq \int_{B(X, 3)}\|f\|_{L^{2}(B(X, 2))}\|f\|_{L^{2}\left(B\left(Y, \frac{1}{2}\right)\right)} d Y .
\end{aligned}
$$

As $B(X, 2) \cap B(Y, 3)=B(X, 2)$ for $Y \in B\left(X, \frac{1}{2}\right)$, we infer that

$$
\frac{\omega_{d}}{2^{d}}\|f\|_{L^{2}(B(X, 2))}^{2} \leq\|f\|_{L^{2}(B(X, 2))} \int_{B(X, 3)}\|f\|_{L^{2}\left(B\left(Y, \frac{1}{2}\right)\right)} d Y,
$$

where $\omega_{d}$ is the volume of the unit ball in $\mathbb{R}^{d}$, and this gives the result.

Now let us prove (2.4). By Sobolev embedding, we have

$$
\left\|\nabla^{k} z_{ \pm}(t)\right\|_{L^{p_{k}(B(X, 1))}} \leq C\left\|z_{ \pm}(t)\right\|_{H^{N}(B(X, 1))} \leq C \rho_{ \pm}(t, X)
$$

where $\frac{1}{p_{k}}=\frac{k-1}{2(N-1)}$ for $1 \leq k \leq N$ and $\frac{1}{p_{k}}=0$ for $k=0$. Thus, for $k+j \leq N+1,0 \leq k, j \leq N$, we have $\frac{1}{p_{k}}+\frac{1}{p_{j}} \leq \frac{1}{2}$ and

$$
\begin{aligned}
\left\|\left|\nabla^{k} z_{+}\left\|\nabla^{j} z_{-} \mid(t)\right\|_{L^{2}(B(X, 1))}\right.\right. & \leq C\left\|\nabla^{k} z_{+}(t)\right\|_{L^{p_{k}(B(X, 1))}}\left\|\nabla^{j} z_{-}(t)\right\|_{L^{p_{j}}(B(X, 1))} \\
& \leq C \rho_{+}(t, X) \rho_{-}(t, X) .
\end{aligned}
$$

Due to $\operatorname{div} z_{ \pm}=0$, we infer from the first equation of (1.3) that

$$
-\triangle p=\partial_{i}\left(z_{+}^{j} \partial_{j} z_{-}^{i}\right)=\partial_{i} z_{+}^{j} \partial_{j} z_{-}^{i}=\partial_{i} \partial_{j}\left(z_{+}^{j} z_{-}^{i}\right) .
$$

Then by the interior elliptic estimates, we get

$$
\begin{aligned}
& \|\nabla p(t)\|_{H^{N}\left(B\left(X, \frac{1}{2}\right)\right)} \leq C\|\nabla p(t)\|_{L^{\infty}(B(X, 1))}+C\|\triangle p(t)\|_{H^{N-1}(B(X, 1))} \\
& \leq C\|\nabla p(t)\|_{L^{\infty}(B(X, 1))}+C \sum_{k+j \leq N-1, k, j \geq 0}\left\|\left|\nabla^{k+1} z_{+}\left\|\nabla^{j+1} z_{-} \mid(t)\right\|_{L^{2}(B(X, 1))}\right.\right. \\
& \leq C\|\nabla p(t)\|_{L^{\infty}(B(X, 1))}+C \rho_{+}(t, X) \rho_{-}(t, X),
\end{aligned}
$$

from which and Lemma 2.1, we infer that

$$
F(t, X) \leq C\|\nabla p(t)\|_{L^{\infty}(B(X, 4))}+C \int_{B(X, 3)} \rho_{+}(t, Y) \rho_{-}(t, Y) d Y .
$$

It remains to estimate $\|\nabla p(t)\|_{L^{\infty}(B(X, 4))}$. For this, we use the following representation formula of the pressure $p(t, X)$ :

$$
\begin{aligned}
-\nabla p(t, X)= & \int_{\mathbb{R}^{d}} \nabla N(X-Y) \theta(|X-Y|) \triangle p(t, Y) d Y \\
& +\int_{\mathbb{R}^{d}} \partial_{i} \partial_{j}(\nabla N(X-Y)(1-\theta(|X-Y|)))\left(z_{+}^{j} z_{-}^{i}\right)(t, Y) d Y,
\end{aligned}
$$

where $N(X)$ is the Newton potential. Thanks to $\left|\nabla^{k} N(X)\right| \leq C|X|^{2-d-k}$ and Sobolev embedding, we obtain

$$
|\nabla p(t, X)| \leq C \int_{B(X, 2)} \frac{d Y}{|X-Y|^{d-1}}\|\triangle p(t)\|_{L^{\infty}(B(X, 2))}+C \int_{\mathbb{R}^{d}} \frac{\left|z_{+}\right|\left|z_{-}\right|(t, Y) d Y}{1+|X-Y|^{d+1}}
$$




$$
\leq C\|\triangle p(t)\|_{H^{N-1}(B(X, 2))}+C \int_{\mathbb{R}^{d}} \frac{\left.\rho_{+}(t, Y) \rho_{-}(t, Y)\right) d Y}{1+|X-Y|^{d+1}} .
$$

Notice that $\|\triangle p(t)\|_{H^{N-1}\left(B\left(Y, \frac{1}{2}\right)\right)} \leq C \rho_{+}(t, Y) \rho_{-}(t, Y)$, which along with Lemma 2.1 gives

$$
\|\triangle p(t)\|_{H^{N-1}(B(X, 2))} \leq C \int_{B(X, 3)} \rho_{+}(t, Y) \rho_{-}(t, Y) d Y .
$$

This shows that

$$
|\nabla p(t, X)| \leq C \int_{\mathbb{R}^{d}} \frac{\left.\rho_{+}(t, Y) \rho_{-}(t, Y)\right) d Y}{1+|X-Y|^{d+1}}
$$

Thanks to $\frac{1}{1+\left|X^{\prime}-Y\right|^{d+1}} \leq \frac{C}{1+|X-Y|^{d+1}}$ for $X^{\prime} \in B(X, 4)$, we have

$$
\|\nabla p(t)\|_{L^{\infty}(B(X, 4))} \leq C \int_{\mathbb{R}^{d}} \frac{\left.\rho_{+}(t, Y) \rho_{-}(t, Y)\right) d Y}{1+|X-Y|^{d+1}}
$$

Inserting this into (2.5), we arrive at (2.4).

\section{COMPARISON PRINCIPLE}

It follows from (2.1) and (2.4) that

$$
\partial_{t} \rho_{ \pm} \mp B_{0} \cdot \nabla \rho_{ \pm}-\mu \triangle \rho_{ \pm} \leq C_{1}\left(\rho_{+} \rho_{-}\right) * N_{1},
$$

where $N_{1}(X)=\left(1+|X|^{d+1}\right)^{-1}$.

To control $\rho_{ \pm}$, we establish the following comparison principle.

Lemma 3.1. Let $0 \leq \rho_{ \pm}^{1} \in L^{\infty} \cap C^{0}\left([0, T) \times \mathbb{R}^{d}\right)$ satisfy

$$
\partial_{t} \rho_{ \pm}^{1} \mp B_{0} \cdot \nabla \rho_{ \pm}^{1}-\mu \triangle \rho_{ \pm}^{1} \geq C_{1}\left(\rho_{+}^{1} \rho_{-}^{1}\right) * N_{1} .
$$

If $\rho_{ \pm}(0) \leq \rho_{ \pm}^{1}(0)$ in $\mathbb{R}^{d}$, then $\rho_{ \pm} \leq \rho_{ \pm}^{1}$ in $[0, T) \times \mathbb{R}^{d}$.

Proof. It follows from (3.1) and (3.2) that

$$
\partial_{t}\left(\rho_{ \pm}-\rho_{ \pm}^{1}\right) \mp B_{0} \cdot \nabla\left(\rho_{ \pm}-\rho_{ \pm}^{1}\right)-\mu \triangle\left(\rho_{ \pm}-\rho_{ \pm}^{1}\right) \leq C_{1}\left(\rho_{+} \rho_{-}-\rho_{+}^{1} \rho_{-}^{1}\right) * N_{1} .
$$

Let $f^{+}=\max (f, 0)$. By maximum principle, we deduce that for $t \in[0, T)$,

$$
\begin{aligned}
\left\|\left(\rho_{ \pm}-\rho_{ \pm}^{1}\right)^{+}(t)\right\|_{L^{\infty}\left(\mathbb{R}^{d}\right)} & \leq \int_{0}^{t} C_{1}\left\|\left(\left(\rho_{+} \rho_{-}-\rho_{+}^{1} \rho_{-}^{1}\right)(s) * N_{1}\right)^{+}\right\|_{L^{\infty}\left(\mathbb{R}^{d}\right)} d s \\
& \leq \int_{0}^{t} C_{1}\left\|\left(\rho_{+} \rho_{-}-\rho_{+}^{1} \rho_{-}^{1}\right)^{+}(s) * N_{1}\right\|_{L^{\infty}\left(\mathbb{R}^{d}\right)} d s \\
& \leq C \int_{0}^{t}\left\|\left(\rho_{+} \rho_{-}-\rho_{+}^{1} \rho_{-}^{1}\right)^{+}(s)\right\|_{L^{\infty}\left(\mathbb{R}^{d}\right)} d s \\
& \leq C M \int_{0}^{t}\left(\left\|\left(\rho_{+}-\rho_{+}^{1}\right)^{+}(s)\right\|_{L^{\infty}\left(\mathbb{R}^{d}\right)}+\left\|\left(\rho_{-}-\rho_{-}^{1}\right)^{+}(s)\right\|_{L^{\infty}\left(\mathbb{R}^{d}\right)}\right) d s
\end{aligned}
$$

where $M=\left\|\rho_{+}+\rho_{-}^{1}\right\|_{L^{\infty}\left([0, T) \times \mathbb{R}^{d}\right)}<+\infty$. This implies that $\left\|\left(\rho_{ \pm}-\rho_{ \pm}^{1}\right)^{+}(t)\right\|_{L^{\infty}\left(\mathbb{R}^{d}\right)}=0$, hence, $\rho_{ \pm}(t) \leq \rho_{ \pm}^{1}(t)$ for $t \in[0, T)$.

To construct comparison functions $\rho_{ \pm}^{1}$, we need the following observation. 
Lemma 3.2. There exists an absolute constant $C_{0}>1$ so that

$$
C_{0}^{-1} N_{1} \leq N_{1} * N_{1} \leq C_{0} N_{1}, \quad \rho_{ \pm}(0) \leq C_{0} \rho_{ \pm}(0) * N_{1} .
$$

Here $\left\|N_{1}\right\|_{L^{1}\left(\mathbb{R}^{d}\right)} \leq C_{0}$.

Proof. As $N_{1}(X) \leq C N_{1}(Y)$ for $|X-Y| \leq 1$, we have

$$
N_{1} * N_{1}(X) \geq \int_{B(X, 1)} N_{1}(Y) N_{1}(X-Y) d Y \geq C^{-1} N_{1}(X) .
$$

Using the fact that

$$
\min \left(N_{1}(Y), N_{1}(X-Y)\right) \leq C N_{1}(X)
$$

we infer that

$$
\begin{aligned}
N_{1} * N_{1}(X) & =\int_{\mathbb{R}^{d}} N_{1}(Y) N_{1}(X-Y) d Y \\
& =\int_{\mathbb{R}^{d}} \min \left(N_{1}(Y), N_{1}(X-Y)\right) \max \left(N_{1}(Y), N_{1}(X-Y)\right) d Y \\
& \leq \int_{\mathbb{R}^{d}} C N_{1}(X)\left(N_{1}(Y)+N_{1}(X-Y)\right) d Y=2 C N_{1}(X)\left\|N_{1}\right\|_{L^{1}\left(\mathbb{R}^{d}\right)},
\end{aligned}
$$

which gives the first inequality.

Using $\left\|z_{ \pm}(0)\right\|_{H^{N}\left(B\left(Y, \frac{1}{2}\right)\right)} \leq C \rho_{ \pm}(0, Y)$ and Lemma 2.1, we deduce that

$$
\rho_{ \pm}(0, X) \leq\left\|z_{ \pm}(0)\right\|_{H^{N}(B(X, 2))} \leq C \int_{B(X, 3)} \rho_{ \pm}(0, Y) d Y \leq C \rho_{ \pm}(0) * N_{1}(X),
$$

which gives the second inequality.

Now let us construct the comparison function.

Lemma 3.3. Let $\rho_{ \pm}^{0} \in L^{1} \cap C(\mathbb{R}), \rho_{ \pm}^{00} \in L^{2} \cap L^{\infty} \cap C\left(\mathbb{R}^{k} \times \mathbb{T}^{d-k}\right)$. Assume that

$$
\begin{aligned}
& 0 \leq \rho_{ \pm}^{00}(x, y) \leq \rho_{ \pm}^{0}(x) \quad \text { for } x \in \mathbb{R}, y \in \mathbb{R}^{d-1}, \\
& C_{0}^{-1} \rho_{ \pm}^{00} \leq \rho_{ \pm}^{00} * N_{1} \leq C_{0} \rho_{ \pm}^{00} .
\end{aligned}
$$

Then there exists $\epsilon_{0}>0$ depending only on $C_{0}, C_{1}$ such that if $\left\|\rho_{ \pm}^{0}\right\|_{L^{1}(\mathbb{R})}<\epsilon_{0}$, then there exists $0 \leq \rho_{ \pm}^{1} \in L^{\infty} \cap C\left([0,+\infty) \times \mathbb{R}^{d}\right)$ satisfying (3.2) and $\rho_{ \pm}^{00} \leq \rho_{ \pm}^{1}(0)$. Moreover,

$$
\left\|\rho_{ \pm}^{1}(t)\right\|_{L^{2}\left(\mathbb{R}^{k} \times \mathbb{T}^{d-k}\right)} \leq C\left\|\rho_{ \pm}^{00}\right\|_{L^{2}\left(\mathbb{R}^{k} \times \mathbb{T}^{d-k}\right)}
$$

Proof. Step 1. Construction of the data

For $\mu=0$, we set $g_{ \pm}^{00}=\rho_{ \pm}^{00}, g_{ \pm}^{0}=\rho_{ \pm}^{0}$. For $\mu>0$, we set

$$
g_{ \pm}^{00}(X)=\frac{1}{2 \mu} \int_{0}^{+\infty} e^{-\frac{y}{2 \mu}} \rho_{ \pm}^{00}\left(X \mp B_{0} y\right) d y, \quad g_{ \pm}^{0}(x)=\frac{1}{2 \mu} \int_{0}^{+\infty} e^{-\frac{y}{2 \mu}} \rho_{ \pm}^{0}(x \mp y) d y .
$$

It is easy to check that

$$
\begin{aligned}
& g_{ \pm}^{0} \pm 2 \mu \partial_{x} g_{ \pm}^{0}=\rho_{ \pm}^{0}, \quad g_{ \pm}^{00} \pm 2 \mu B_{0} \cdot \nabla g_{ \pm}^{00}=\rho_{ \pm}^{00} \\
& \left\|g_{ \pm}^{0}\right\|_{L^{1}(\mathbb{R})}=\left\|\rho_{ \pm}^{0}\right\|_{L^{1}(\mathbb{R})}, \quad\left\|g_{ \pm}^{00}\right\|_{L^{\infty}\left(\mathbb{R}^{d}\right)} \leq\left\|\rho_{ \pm}^{00}\right\|_{L^{\infty}\left(\mathbb{R}^{d}\right)},
\end{aligned}
$$

and $0 \leq g_{ \pm}^{00}(x, y) \leq g_{ \pm}^{0}(x)$ for $x \in \mathbb{R}, y \in \mathbb{R}^{d-1}$.

Let

$$
h_{ \pm}^{0}(x)=\frac{1}{2 \epsilon_{0}} \int_{0}^{+\infty}\left(\rho_{ \pm}^{0}+g_{ \pm}^{0}\right)(x \mp y) d y
$$


Then we have

$$
0 \leq h_{ \pm}^{0}<1, \quad \pm \partial_{x} h_{ \pm}^{0}=\left(\rho_{ \pm}^{0}+g_{ \pm}^{0}\right) /\left(2 \epsilon_{0}\right) .
$$

Step 2. Construction of comparison function Let $\left(\rho_{ \pm}^{01}, g_{ \pm}^{01}\right)(t, X)$ be the solution to

$$
\partial_{t} f \mp B_{0} \cdot \nabla f-\mu \triangle f=0, \quad f(0, X)=\left(\rho_{ \pm}^{00}, g_{ \pm}^{00}\right)(X),
$$

and $\left(\rho_{ \pm}^{11}, g_{ \pm}^{1}, h_{ \pm}^{1}\right)(t, x)$ be the solution to

$$
\partial_{t} f \mp \partial_{x} f-\mu \partial_{x}^{2} f=0, \quad f(0, x)=\left(\rho_{ \pm}^{0}, g_{ \pm}^{0}, h_{ \pm}^{0}\right)(x) .
$$

Thanks to the construction of the data, we find that

$$
\begin{aligned}
& 0 \leq h_{ \pm}^{1}<1, \quad \pm \partial_{x} h_{ \pm}^{1}=\left(\rho_{ \pm}^{11}+g_{ \pm}^{1}\right) /\left(2 \epsilon_{0}\right) \\
& g_{ \pm}^{01} \pm 2 \mu B_{0} \cdot \nabla g_{ \pm}^{01}=\rho_{ \pm}^{01}, \\
& 0 \leq \rho_{ \pm}^{01}(t, x, y) \leq \rho_{ \pm}^{11}(t, x), \quad 0 \leq g_{ \pm}^{01}(t, x, y) \leq g_{ \pm}^{1}(t, x) .
\end{aligned}
$$

Now we take $\rho_{ \pm}^{1}(t)=C_{0} \rho_{ \pm}^{10}(t) * N_{1}$, where

$$
\rho_{ \pm}^{10}(t, X)=\rho_{ \pm}^{01}(t, X)+g_{ \pm}^{01}(t, X) h_{\mp}^{1}(t, x) .
$$

Step 3. Verification of the conditions

By our construction, it is easy to check that

$$
\begin{aligned}
\partial_{t} & \rho_{ \pm}^{10} \mp B_{0} \cdot \nabla \rho_{ \pm}^{10}-\mu \triangle \rho_{ \pm}^{10}=\left(\partial_{t} \rho_{ \pm}^{00} \mp B_{0} \cdot \nabla \rho_{ \pm}^{00}-\mu \triangle \rho_{ \pm}^{00}\right) \\
& +\left(\partial_{t} g_{ \pm}^{01} \mp B_{0} \cdot \nabla g_{ \pm}^{01}-\mu \triangle g_{ \pm}^{01}\right) h_{\mp}^{1}+g_{ \pm}^{01}\left(\partial_{t} h_{\mp}^{1} \mp \partial_{x} h_{\mp}^{1}-\mu \partial_{x}^{2} h_{\mp}^{1}\right) \\
& -2 \mu B_{0} \cdot \nabla g_{ \pm}^{01} \partial_{x} h_{\mp}^{1} \\
= & 0+0 \mp 2 g_{ \pm}^{01} \partial_{x} h_{\mp}^{1}-2 \mu B_{0} \cdot \nabla g_{ \pm}^{01} \partial_{x} h_{\mp}^{1} \\
= & \mp\left(2 g_{ \pm}^{01} \pm 2 \mu B_{0} \cdot \nabla g_{ \pm}^{01}\right) \partial_{x} h_{\mp}^{1} \\
= & \left(g_{ \pm}^{01}+\rho_{ \pm}^{01}\right)\left(\rho_{\mp}^{11}+g_{\mp}^{1}\right) /\left(2 \epsilon_{0}\right) \geq\left(g_{ \pm}^{01}+\rho_{ \pm}^{01}\right)\left(\rho_{\mp}^{01}+g_{\mp}^{01}\right) /\left(2 \epsilon_{0}\right),
\end{aligned}
$$

which implies that

$$
\partial_{t} \rho_{ \pm}^{1} \mp B_{0} \cdot \nabla \rho_{ \pm}^{1}-\mu \triangle \rho_{ \pm}^{1} \geq C_{0} /\left(2 \epsilon_{0}\right)\left(\left(g_{+}^{01}+\rho_{+}^{01}\right)\left(\rho_{-}^{01}+g_{-}^{01}\right)\right) * N_{1} .
$$

As $0 \leq h_{ \pm}^{1}<1$, we have

$$
\rho_{ \pm}^{1}(t) \leq C_{0} \rho_{ \pm}^{01}(t) * N_{1}+C_{0} g_{ \pm}^{01}(t) * N_{1} .
$$

Since $\rho_{ \pm}^{01}(t)$ and $\rho_{ \pm}^{01}(t) * N_{1}$ satisfy $\partial_{t} f \mp B_{0} \cdot \nabla f-\mu \triangle f=0$ and $\rho_{ \pm}^{01}(0) * N_{1}=\rho_{ \pm}^{00} * N_{1} \leq$ $C_{0} \rho_{ \pm}^{00}=C_{0} \rho_{ \pm}^{01}(0)$, we conclude that $\rho_{ \pm}^{01}(t) * N_{1} \leq C_{0} \rho_{ \pm}^{01}(t)$. Thanks to $g_{ \pm}^{00} * N_{1} \leq C_{0} g_{ \pm}^{00}$, we similarly have $g_{ \pm}^{01}(t) * N_{1} \leq C_{0} g_{ \pm}^{01}(t)$. Thus,

$$
0 \leq \rho_{ \pm}^{1}(t) \leq C_{0}^{2}\left(\rho_{ \pm}^{01}(t)+g_{ \pm}^{01}(t)\right)
$$

which along with (3.3) gives

$$
\partial_{t} \rho_{ \pm}^{1} \mp B_{0} \cdot \nabla \rho_{ \pm}^{1}-\mu \triangle \rho_{ \pm}^{1} \geq\left(\rho_{+}^{1} \rho_{-}^{1}\right)(t) * N_{1} /\left(2 \epsilon_{0} C_{0}^{3}\right) .
$$

Taking $\epsilon_{0}=1 /\left(2 C_{0}^{3} C_{1}\right)$, we find that $0 \leq \rho_{ \pm}^{1} \in L^{\infty} \cap C\left([0,+\infty) \times \mathbb{R}^{d}\right)$ satisfies (3.2). As $\rho_{ \pm}^{10}(0) \geq \rho_{ \pm}^{01}(0)=\rho_{ \pm}^{00}$, we have $\rho_{ \pm}^{1}(0)=C_{0} \rho_{ \pm}^{10}(0) * N_{1} \geq C_{0} \rho_{ \pm}^{00} * N_{1} \geq \rho_{ \pm}^{00}$.

By standard energy estimate, we can deduce that

$$
\begin{aligned}
\left\|\rho_{ \pm}^{01}(t)\right\|_{L^{2}\left(\mathbb{R}^{k} \times \mathbb{T}^{d-k}\right)} & \leq\left\|\rho_{ \pm}^{01}(0)\right\|_{L^{2}\left(\mathbb{R}^{k} \times \mathbb{T}^{d-k}\right)}=\left\|\rho_{ \pm}^{00}\right\|_{L^{2}\left(\mathbb{R}^{k} \times \mathbb{T}^{d-k}\right)} \\
\left\|g_{ \pm}^{01}(t)\right\|_{L^{2}\left(\mathbb{R}^{k} \times \mathbb{T}^{d-k}\right)} & \leq\left\|g_{ \pm}^{00}\right\|_{L^{2}\left(\mathbb{R}^{k} \times \mathbb{T}^{d-k}\right)}
\end{aligned}
$$


For $\mu=0,\left\|g_{ \pm}^{00}\right\|_{L^{2}\left(\mathbb{R}^{k} \times \mathbb{T}^{d-k}\right)}=\left\|\rho_{ \pm}^{00}\right\|_{L^{2}\left(\mathbb{R}^{k} \times \mathbb{T}^{d-k}\right)}$, and for $\mu>0$

$$
\begin{aligned}
& \left\|g_{ \pm}^{00}\right\|_{L^{2}\left(\mathbb{R}^{k} \times \mathbb{T}^{d-k}\right)} \leq \frac{1}{2 \mu} \int_{0}^{+\infty} e^{-\frac{y}{2 \mu}}\left\|\rho_{ \pm}^{00}\left(\cdot \mp B_{0} y\right)\right\|_{L^{2}\left(\mathbb{R}^{k} \times \mathbb{T}^{d-k}\right)} d y \\
& =\frac{1}{2 \mu} \int_{0}^{+\infty} e^{-\frac{y}{2 \mu}}\left\|\rho_{ \pm}^{00}\right\|_{L^{2}\left(\mathbb{R}^{k} \times \mathbb{T}^{d-k}\right)} d y=\left\|\rho_{ \pm}^{00}\right\|_{L^{2}\left(\mathbb{R}^{k} \times \mathbb{T}^{d-k}\right)} .
\end{aligned}
$$

Thus, we obtain

$$
\begin{aligned}
\left\|\rho_{ \pm}^{1}(t)\right\|_{L^{2}\left(\mathbb{R}^{k} \times \mathbb{T}^{d-k}\right)} & \leq C_{0}^{2}\left(\left\|\rho_{ \pm}^{01}(t)\right\|_{L^{2}\left(\mathbb{R}^{k} \times \mathbb{T}^{d-k}\right)}+\left\|g_{ \pm}^{01}(t)\right\|_{L^{2}\left(\mathbb{R}^{k} \times \mathbb{T}^{d-k}\right)}\right) \\
& \leq C_{0}^{2}\left(\left\|\rho_{ \pm}^{00}\right\|_{L^{2}\left(\mathbb{R}^{k} \times \mathbb{T}^{d-k}\right)}+\left\|g_{ \pm}^{00}\right\|_{L^{2}\left(\mathbb{R}^{k} \times \mathbb{T}^{d-k}\right)}\right) \\
& \leq 2 C_{0}^{2}\left\|\rho_{ \pm}^{00}\right\|_{L^{2}\left(\mathbb{R}^{k} \times \mathbb{T}^{d-k}\right)}
\end{aligned}
$$

This completes the proof.

\section{Proof of Theorem 1.1}

By the local well-posedness result, there exists a unique solution $z_{ \pm} \in C\left(\left[0, T^{*}\right), H^{N}\left(\mathbb{R}^{k} \times\right.\right.$ $\left.\mathbb{T}^{d-k}\right)$ ) to the MHD equations (1.3), where $T^{*}$ is the maximal existence time of the solution.

Let $\rho_{ \pm}^{00}=C_{0} \rho_{ \pm}(0) * N_{1}$. Then Lemma 3.2 ensures that

$$
\rho_{ \pm}(0) \leq \rho_{ \pm}^{00}, \quad C_{0}^{-1} \rho_{ \pm}^{00} \leq \rho_{ \pm}^{00} * N_{1} \leq C_{0} \rho_{ \pm}^{00} .
$$

Let

$$
\rho_{ \pm}^{0}(x)=C_{0} \int_{\mathbb{R}} \int_{\mathbb{R}^{d-1}} \rho_{ \pm}^{*}\left(x-x^{\prime}\right) N_{1}\left(x^{\prime}, y^{\prime}\right) d y^{\prime} d x^{\prime},
$$

where $\rho_{ \pm}^{*}(x)=\sup _{y \in \mathbb{R}^{d-1}} \rho_{ \pm}(0, x, y)$, thus $J_{ \pm}(0)=\left\|\rho_{ \pm}^{*}\right\|_{L^{1}(\mathbb{R})}$. Thanks to

$$
\rho_{ \pm}^{00}(x, y)=C_{0} \int_{\mathbb{R}} \int_{\mathbb{R}^{d-1}} \rho_{ \pm}\left(0, x-x^{\prime}, y-y^{\prime}\right) N_{1}\left(x^{\prime}, y^{\prime}\right) d y^{\prime} d x^{\prime},
$$

we find that $\rho_{ \pm}^{00}(x, y) \leq \rho_{ \pm}^{0}(x)$ and

$$
\begin{aligned}
\left\|\rho_{ \pm}^{0}\right\|_{L^{1}(\mathbb{R})} & =C_{0} \int_{\mathbb{R}} \int_{\mathbb{R}} \int_{\mathbb{R}^{d-1}} \rho_{ \pm}^{*}\left(x-x^{\prime}\right) N_{1}\left(x^{\prime}, y^{\prime}\right) d y^{\prime} d x^{\prime} d x \\
& =C_{0}\left\|\rho_{ \pm}^{*}\right\|_{L^{1}(\mathbb{R})} \int_{\mathbb{R}} \int_{\mathbb{R}^{d-1}} N_{1}\left(x^{\prime}, y^{\prime}\right) d y^{\prime} d x^{\prime} \\
& =C_{0} J_{ \pm}(0)\left\|N_{1}\right\|_{L^{1}\left(\mathbb{R}^{d}\right)} \leq C_{0}^{2} \epsilon_{1}<\epsilon_{0},
\end{aligned}
$$

if $J_{ \pm}(0) \leq \epsilon_{1}=\epsilon_{0} / 2 C_{0}^{2}$.

Now, Lemma 3.3 ensures that there exists $0 \leq \rho_{ \pm}^{1} \in L^{\infty} \cap C\left([0,+\infty) \times \mathbb{R}^{d}\right)$, which satisfies (3.2), and $\rho_{ \pm}(0) \leq \rho_{ \pm}^{00} \leq \rho_{ \pm}^{1}(0)$ in $\mathbb{R}^{d}$, and

$$
\left\|\rho_{ \pm}^{1}(t)\right\|_{L^{2}\left(\mathbb{R}^{k} \times \mathbb{T}^{d-k}\right)} \leq C\left\|\rho_{ \pm}^{00}\right\|_{L^{2}\left(\mathbb{R}^{k} \times \mathbb{T}^{d-k}\right)} \leq C\left\|\rho_{ \pm}(0)\right\|_{L^{2}\left(\mathbb{R}^{k} \times \mathbb{T}^{d-k}\right)} \leq C\left\|z_{ \pm}(0)\right\|_{H^{N}\left(\mathbb{R}^{k} \times \mathbb{T}^{d-k}\right)} .
$$

Then we infer from Lemma 3.1 that $0 \leq \rho_{ \pm} \leq \rho_{ \pm}^{1}$ in $[0, T) \times \mathbb{R}^{d}$ for $0<T<T^{*}$. Hence, $\left\|z_{ \pm}(t)\right\|_{H^{N}\left(\mathbb{R}^{k} \times \mathbb{T}^{d-k}\right)} \leq C\left\|\rho_{ \pm}(t)\right\|_{L^{2}\left(\mathbb{R}^{k} \times \mathbb{T}^{d-k}\right)} \leq C\left\|\rho_{ \pm}^{1}(t)\right\|_{L^{2}\left(\mathbb{R}^{k} \times \mathbb{T}^{d-k}\right)} \leq C\left\|z_{ \pm}(0)\right\|_{H^{N}\left(\mathbb{R}^{k} \times \mathbb{T}^{d-k}\right)}$ for any $t \in[0, T)$, which implies $T^{*}=+\infty$. 


\section{Decay estimates}

In this section, we provide some decay estimates of the solution in time when $\mu>0$. Here we use the notations in section 3 and section 4 .

Using the fact that $\left(\rho_{ \pm}^{01}, g_{ \pm}^{01}\right)(t, X)=e^{\mu t \triangle}\left(\rho_{ \pm}^{00}, g_{ \pm}^{00}\right)\left(X-B_{0} t\right)$, we have

$$
g_{ \pm}^{01}(t, X)=\frac{1}{2 \mu} \int_{0}^{+\infty} e^{-\frac{y}{2 \mu}} \rho_{ \pm}^{01}\left(t, X \mp B_{0} y\right) d y
$$

which gives

$$
\left\|g_{ \pm}^{01}(t)\right\|_{L^{p}\left(\mathbb{R}^{k} \times \mathbb{T}^{d-k}\right)} \leq\left\|\rho_{ \pm}^{01}(t)\right\|_{L^{p}\left(\mathbb{R}^{k} \times \mathbb{T}^{d-k}\right)}=\left\|e^{\mu t \triangle} \rho_{ \pm}^{00}\right\|_{L^{p}\left(\mathbb{R}^{k} \times \mathbb{T}^{d-k}\right)}
$$

Thus, we deduce that for $2 \leq p \leq \infty$,

$$
\left\|\rho_{ \pm}^{1}(t)\right\|_{L^{p}\left(\mathbb{R}^{k} \times \mathbb{T}^{d-k}\right)} \leq C\left\|e^{\mu t \triangle} \rho_{ \pm}^{00}\right\|_{L^{p}\left(\mathbb{R}^{k} \times \mathbb{T}^{d-k}\right)} .
$$

which along with the fact that $e^{\mu t \triangle} \rho_{ \pm}^{00}=C_{0} e^{\mu t \triangle} \rho_{ \pm}(0) * N_{1}$, we infer that

$$
\begin{aligned}
\left\|\rho_{ \pm}^{1}(t)\right\|_{L^{p}\left(\mathbb{R}^{k} \times \mathbb{T}^{d-k}\right)} & \leq C\left\|e^{\mu t \triangle} \rho_{ \pm}^{00}\right\|_{L^{p}\left(\mathbb{R}^{k} \times \mathbb{T}^{d-k}\right)} \\
& \leq C\left\|e^{\mu t \triangle} \rho_{ \pm}(0)\right\|_{L^{p}\left(\mathbb{R}^{k} \times \mathbb{T}^{d-k}\right)}\left\|N_{1}\right\|_{L^{1}\left(\mathbb{R}^{d}\right)}
\end{aligned}
$$

for $2 \leq p \leq \infty$. Thus, we obtain

$$
\begin{aligned}
\left\|z_{ \pm}(t)\right\|_{W^{1, \infty}\left(\mathbb{R}^{k} \times \mathbb{T}^{d-k}\right)} & \leq C\left\|\rho_{ \pm}^{1}(t)\right\|_{L^{\infty}\left(\mathbb{R}^{k} \times \mathbb{T}^{d-k}\right)} \leq C\left\|e^{\mu t \triangle} \rho_{ \pm}(0)\right\|_{L^{\infty}\left(\mathbb{R}^{k} \times \mathbb{T}^{d-k}\right)} \\
& \leq C(1+\mu t)^{-\frac{k}{4}}\left\|z_{ \pm}(0)\right\|_{H^{N}\left(\mathbb{R}^{k} \times \mathbb{T}^{d-k}\right)} .
\end{aligned}
$$

If $\rho_{ \pm}(0) \in L^{1}\left(\mathbb{R}^{d}\right)$, we similarly have

$$
\begin{aligned}
& \left\|z_{ \pm}(t)\right\|_{H^{N}\left(\mathbb{R}^{k} \times \mathbb{T}^{d-k}\right)} \leq C(1+\mu t)^{-\frac{k}{4}} \\
& \left\|z_{ \pm}(t)\right\|_{W^{1, \infty}\left(\mathbb{R}^{k} \times \mathbb{T}^{d-k}\right)} \leq C(1+\mu t)^{-\frac{k}{2}} .
\end{aligned}
$$

\section{ACKNOWLEDGEMENTS}

This work was supported by NSF of China under Grant 11425103.

\section{REFERENCES}

[1] Abidi H, Zhang P. On the global solution of 3-D MHD system with initial data near equilibrium. Comm. Pure Appl. Math., online.

[2] Bardos C, Sulem C, Sulem P.-L. Longtime dynamics of a conductive fluid in the presence of a strong magnetic field. Trans. Amer. Math. Soc., 1988, 305:175-191.

[3] Califano F, Chiuderi C. Resistivity-independent dissipation of magnetrodydrodynamic waves in an inhomogeneous plasma. Phy. Rev. E, 1999, part B 60: 4701-4707.

[4] Cai Y, Lei Z. Global Well-posedness of the Incompressible Magnetohydrodynamics. arXiv:1605.00439

[5] Cao C, Wu J. Global regularity for the 2D MHD equations with mixed partial dissipation and magnetic diffusion. Adv. Math., 2011, 226: 1803-1822.

[6] He L, Xu L,Yu P. On global dynamics of three dimensional magnetohydrodynamics: nonlinear stability of Alfvén waves. arXiv:1603.08205.

[7] Lin F, Xu L., Zhang P. Global small solutions of 2-D incompressible MHD system. J. Differential Equations, 2015, 259: 5440-5485.

[8] Ren X, Wu J, Xiang Z, Zhang Z. Global existence and decay of smooth solution for the 2-D MHD equations without magnetic diffusion. J. Funct. Anal., 2014, 267: 503-541.

[9] Ren X, Xiang Z, Zhang Z. Global well-posedness for the 2D MHD equations without magnetic diffusion in a strip domain. Nonlinearity, 2016, 29:1257-1291.

[10] Wei D, Zhang Z. Global well-posedness of the MHD equations in a homogeneous magnetic field. Analysis \& PDE. 
[11] Zhang T. An elementary proof of the global existence and uniqueness theorem to 2-D incompressible nonresistive MHD system. arXiv:1404.5681.

School of Mathematical Sciences, Peking University, 100871, Beijing, P. R. China

E-mail address: jnwdyi@163.com

School of Mathematical Sciences, Peking University, 100871, Beijing, P. R. China

E-mail address: zfzhang@math.pku.edu.cn 